\title{
Online Uniformly Inserting Points on Grid
}

\author{
Yong Zhang ${ }^{1}$, Zhuo Chang ${ }^{2}$, Francis Y.L. Chin ${ }^{1 \star}$, \\ Hing-Fung Ting ${ }^{1 \star \star}$, and Yung H. Tsin ${ }^{3 \star \star \star}$ \\ 1 Department of Computer Science, The University of Hong Kong, Hong Kong \\ \{yzhang, chin, hfting\}@cs.hku.hk \\ 2 College of Mathematics and Computer Science, Hebei University, China. \\ changzhuo@cmc.hbu.cn \\ 3 School of Computer Science, University of Windsor, Canada \\ peter@uwindsor.ca
}

\begin{abstract}
In this paper, we consider the problem of inserting points in a square grid, which has many background applications, including halftone in reprographic and image processing. We consider an online version of this problem, i.e., the points are inserted one at a time. The objective is to assign the points as uniform as possible. Precisely speaking, after each insertion, the gap ratio should be as small as possible. In this paper, we give an insertion strategy with the maximal gap ratio no more than $2 \sqrt{2} \approx 2.828$, which is the first result on uniformly inserting point on grid. Moreover, we show that no online algorithm can achieve the maximal gap ratio strictly less than 2.5 for a $3 \times 3$ grid.
\end{abstract}

\section{Introduction}

In this paper, we consider the problem of online inserting points in a square grid such that the distribution of the inserted points is as uniform as possible. In the real world, there are many applications needing the uniform distribution for some value in a given area, e.g., halftone, distribution of chain stores in an area.

Halftone is a very important technique in image processing, which simulates the actual continuous image by discrete dots such that in the view of human's eyes, this kind of simulation is almost same as the original image. To achieve better performances, e.g., higher resolutions, dithering method [3] is often applied in halftone. One of the most important parts in dithering is how to generate the dither matrix, on which the quality of simulation heavily depends. Each element in the dither matrix represents a threshold value of the grey level between black and white. For example, let's consider the dither matrix in Figure 1, an absolutely dark spot with the grey level of 32 will be able to meet all the threshold values of the dither matrix, thus, all the 64 elements (pixels) will be black; similarly, a grey level is 0 will have all white pixels. As for any of the remaining grey level $x$, only those matrix elements (pixels) whose values (thresholds) are equal to or below $x$ will turn black, e.g., for grey level $=10$, only 10 elements (pixels) are black and these elements have to be distributed uniformly inside the matrix. Since the uniformity has to be applied to all grey levels, this reduces to our problem which is online inserting points uniformly in a square grid. Figure 1 gives an example of

\footnotetext{
* Research supported by HK RGC grant HKU-7113/07E

${ }^{\star \star}$ Research supported by HK RGC grant HKU-7171/08E

$\star \star \star$ Research supported by NSERC under grant NSERC 7811-2009
} 
a dither matrix in [3] and the simulation depends on this matrix. Formally, the dither matrix is a $n \times n$ matrix, the value of each element is from 0 to $n^{2}-1$, and the values up to each $i\left(0 \leq i \leq n^{2}-1\right)$ are uniformly distributed.

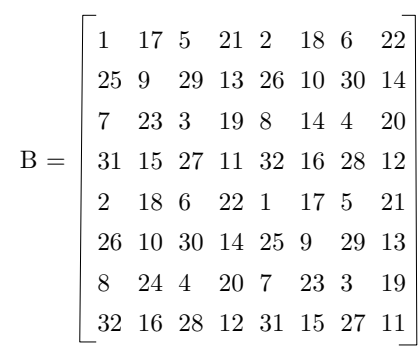

(a) dither matrix

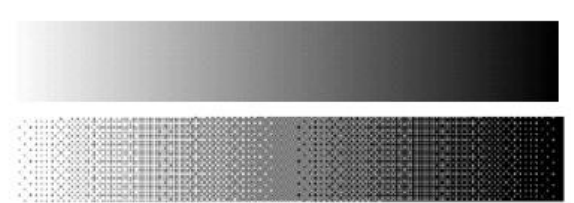

(b) original graph and its simulation

Fig. 1. A simulation based on a dither matrix

Another motivation is the distribution of chain stores in an area. A famous chain store has planned to establish its business in a district by establishing a number of stores at the road junctions in a city with Manhattan-like road network one by one. Assume the clients are distributed uniformly, and each client will be served by its nearest store. In order to minimize the unnecessary competition among its own stores, the established stores at any time should be distributed as uniformly as possible while the stores once established cannot be dismantled or relocated.

In this problem, we consider the insertion of the points in an online manner, i.e., the points are inserted one by one, and the algorithm does not know the number of inserted points in advance. After the insertion of each point, the uniformity is guaranteed, the uniformity is a measurement of how uniform the inserted points distributed. There are several ways to define the uniformity of a set of points. Some studies define the uniformity by the minimal pairwise distance $[5,7]$. In discrepancy theory $[4,6]$, the uniformity is defined by the ratio between the maximal and minimal number of points in a fixed shape within the area. In this paper, the uniformity is defined by the gap ratio, i.e., the ratio between the maximal gap (the diameter of the largest empty circle) and the minimal gap (the minimal pairwise distance).

\section{Problem Statement}

Let $\mathcal{S}^{2}$ be an $m \times m$ unit square grid in the 2-dimensional square $\mathcal{R}^{2}$ such that the four corners of $\mathcal{S}^{2}$ and $\mathcal{R}^{2}$ are located at the same position. Let $\mathcal{P}=\{1,2, \ldots, n\}$ be a point request sequence. In the initial state, the four corner grid positions in $\mathcal{S}^{2}$ are assigned. Each request must be assigned on some grid position in $\mathcal{S}^{2}$, and each grid position can satisfy at most one request, thus $n \leq(m+1)^{2}-4$. Denote $p_{i}$ to be the grid position in satisfying the $i$-th request, and $\mathcal{S}_{i}=\left\{p_{1}, \ldots, p_{i}\right\} \cup \mathcal{S}_{0}$ is the configuration in $\mathcal{S}^{2}$ after inserting the $i$-th point, where $\mathcal{S}_{0}$ consists of the four corner points of $\mathcal{S}^{2}$.

Define the maximal gap at step $i$ to be $G_{i}=\max _{p \in \mathcal{R}^{2}} \min _{q \in \mathcal{S}_{i}} 2 d(p, q)$, the minimal gap at step $i$ to be $g_{i}=\min _{p, q \in \mathcal{S}_{i}, p \neq q} d(p, q)$, where $d(\cdot, \cdot)$ is the Euclidean 
distance, and define the $i$-th gap ratio as $r_{i}=G_{i} / g_{i}$. The maximum gap and the minimum gap imply the diameter of the largest empty circle ${ }^{4}$ and the minimum pairwise distance, respectively.

The objective of this problem is assigning points into the grid as uniform as possible, i.e., minimize the maximal gap ratio $\left(\min \max _{i} r_{i}\right)$ after each insertion.

For the $m \times m$ square grid, let $(0,0)$ represent its up-left-most point $a$. Each grid point $p$ can be represented by $(i, j)$, where $i$ is the difference between the $x$-coordinate of $a$ and $p, j$ is the difference between the $y$-coordinate of $a$ and $p$. We say a square or rectangle is of size $i \times j$ if the lengths of two connected edges of the square or rectangle are $i$ and $j$ respectively. Let $R$ be the circumradius of a triangle $U V W$, we have $|R|=\frac{u v w}{4 \Delta}$, where $u, v$ and $w$ are the length of edges of the triangle and $\Delta$ is the area of the triangle.

Now we give an example to show the maximal gap, minimal gap, and gap ratio. At the initial state, there are only four assigned points at the four corners $a, b, c$ and $d$ as shown in Fig. 2, the maximal gap $G_{0}=\sqrt{2} \cdot m$ while the minimal gap $g_{0}=m$, the gap ratio $r_{0}=\sqrt{2}$. If the first point $p_{1}$ is inserted at the center of the square, the current maximal gap $G_{1}=m$ while the minimal gap $g_{1}=\sqrt{2} \cdot m / 2$ and the gap ratio $r_{1}=\sqrt{2}$.

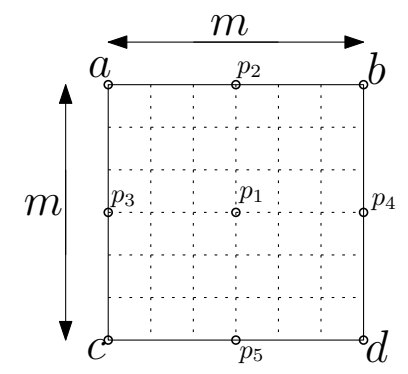

Fig. 2. An insertion of five points in a grid.

\section{Related Works:}

Uniformly inserting points in a given area is studied in some papers. If the points can be inserted at any position in the given area, Teramoto et al [8] and Asano et al [2] showed that the greedy algorithm (voronoi insertion) has uniformity 2 . In one dimensional case, if the algorithm knows the number $n$ of the inserted points, an insertion strategy with maximal gap ratio $2^{\lfloor n / 2\rfloor /(\lfloor n / 2\rfloor+1)}$, which is slightly less than 2, can be achieved. Moreover, they gave a local search heuristic for uniformly inserting points on two dimensional square, the experimental results showed that the maximal gap ratio is less than 2 if the number of inserted points is not large. If the point must be assigned at grid point, Asano [1] gave an insertion strategy with uniformity 2 for one dimensional case.

\section{Our Contributions:}

To uniformly insert the points into a square, an intuitive idea is to insert each

\footnotetext{
${ }^{4}$ Since we only focus on the area $\mathcal{R}^{2}$, the center of the largest empty circle must be within $\mathcal{R}^{2}$.
} 
point at the center of the largest empty circumcircle of a triangle within the square. But this idea is not a good strategy when implementing on the square grid. For example, consider a $6 \times 6$ square grid as shown in Figure 2, if each point is inserted at the center of the largest empty circumcircle, the first 5 points must be inserted as shown in Figure 2. No matter where the next point is inserted, the gap ratio will be no less than 3 .

In the following part of this paper, we give an insertion strategy for the problem of inserting points in a square grid with the maximal gap ratio no more than $2 \sqrt{2} \approx 2.828$. For the problem of uniformly inserting points, this is the first result on inserting points at grid position. Moreover, we show that no online inserting strategy can hold the maximal gap ratio strictly less than 2.5 for $3 \times 3$ grid.

\section{Inserting Method}

Inserting each point at the center of the largest empty circle is a good strategy if the size of the grid is some power of 2, i.e., $m=2^{k}$. In this case, insertion at the center can always hold the gap ratio to no more than 2. Another observation is that once a point is inserted, the grid will be somewhat partitioned into regions which can be handled independently and locally. In the following, we devise our heuristic based on these observations, to achieve good performance. Instead of inserting each point at the center of the largest empty circle, we choose a proper position which partitions the grid into several parts: some of them are square grids whose sizes are some power of 2 ; some are square grids with sizes similar to the above ones; the others are rectangles with sizes between the above two types of square grids. Assigning the point at such position can guarantee the maximal gap ratio is not large.

Our strategy is done phase by phase. The order of inserting points on some grid positions is based on the current configuration of assigned points. At the begining and end of each phase, the square grid can be partitioned into four disjoint parts: the up-left part is the combination of small square grids of the same size $2^{k^{\prime}} \times 2^{k^{\prime}}$; the down-right part is a square grid of size $m^{\prime} \times m^{\prime}$; the up-right and down-left parts are combinations of rectangle grids of size $2^{k^{\prime}} \times m^{\prime}$, as shown in Figure 3(a).

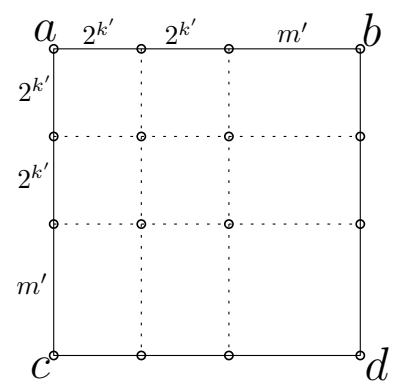

(a) before phase $i$

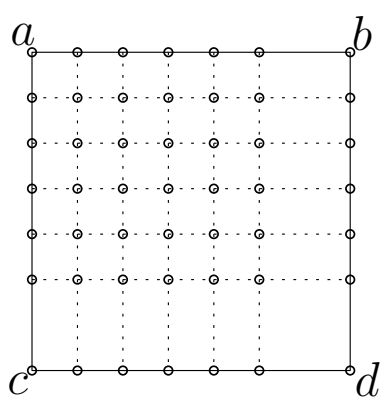

(b) after phase $i$

Fig. 3. the configuration of the square grid before and after a phase of insertion

When starting to insert points in a phase, if the small square grids of the up-left part are larger than the down-right square, we insert some points into the 
center at each small square grids; otherwise, the down-right part is larger, then we insert points into the down-right square. According to the following insertion strategy, we can insert some points such that either the size of the small square grid in the up-left part is decreased, or the up-left part is enlarged. From the analysis of the strategy, the gap ratio is bounded by $2 \sqrt{2}$ after each insertion. The configuration after a phase of insertion from the configuration in Figure 3(a) is shown in Figure 3(b).

\subsection{The First Phase}

In the initial state, there are only four assigned points located at the four corners of the square grid. To assign the first point, we must determine the $(x, y)$-coordinate for $p_{1}$. Find the integer $k$ such that $3 \cdot 2^{k-1} \leq m<3 \cdot 2^{k}$. Insert the first point $p_{1}$ at $\left(2^{k}, 2^{k}\right)$.

Case 1: $3 \cdot 2^{k-1} \leq m<(2+\sqrt{2}) \cdot 2^{k-1}$

In this case, we insert $p_{2}$ at $\left(2^{k-1}, 2^{k-1}\right), p_{3}$ at $\left(2^{k}, 0\right), p_{4}$ at $\left(0,2^{k}\right), p_{5}$ until $p_{12}$ are assigned as shown in Figure 4 .

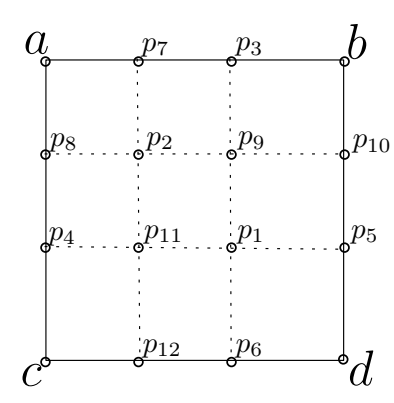

Fig. 4. Case 1 of the first phase.

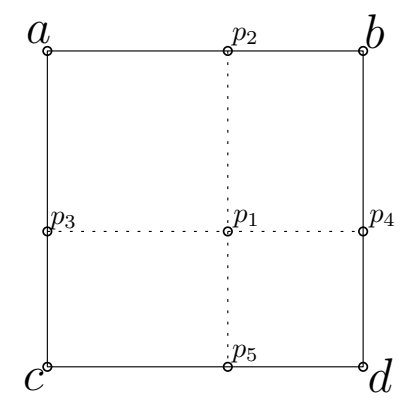

Fig. 5. Case 2 of the first phase.

Let $m=(1+x) \times 2^{k}$, we have $1 / 2 \leq x<\sqrt{2} / 2$. Now we analyze the gap ratio after each insertion.

Lemma 1 In case $1: 3 \cdot 2^{k-1} \leq m<(2+\sqrt{2}) \cdot 2^{k-1}$, the gap ratio is no more than $2 \sqrt{2}$ after each insertion according to the strategy. (Proof is in Appendix.)

After this insertion phase, the up-left part is the combination of small square grids of size $2^{k-1} \times 2^{k-1}$, the down-right part is a square grid of size $\left(m-2^{k}\right) \times$ $\left(m-2^{k}\right)$, the up-right and down-left parts are combinations of rectangles of size $2^{k-1} \times\left(m-2^{k}\right)$.

Case 2: $(2+\sqrt{2}) \cdot 2^{k-1} \leq m<(2+1 / \sqrt{2}) \cdot 2^{k}$

In this case, insert $p_{2}$ at $\left(2^{k}, 0\right), p_{3}$ at $\left(0,2^{k}\right), p_{4}$ at $\left(m, 2^{k}\right)$, and $p_{5}$ at $\left(2^{k}, m\right)$, which are shown in Figure 5 .

Let $m=(1+x) 2^{k}$, we have $\sqrt{2} / 2 \leq x \leq 1+\sqrt{2} / 2$. Now we analyze the gap ratio after each insertion. 
Lemma 2 In case 2: $(2+\sqrt{2}) \cdot 2^{k-1} \leq m<(2+1 / \sqrt{2}) \cdot 2^{k}$, the gap ratio is no more than $2 \sqrt{2}$ after each insertion according to the strategy. (Proof is in Appendix.)

After this phase, the up-left part is a square grid of size $\left(2^{k}, 2^{k}\right)$, the downright part is a square grid of size $\left(m-2^{k}, m-2^{k}\right)$, the up-right and down-left parts are rectangles of size $\left(2^{k}, m-2^{k}\right)$.

Case 3: $(2+1 / \sqrt{2}) \cdot 2^{k} \leq m<3 \cdot 2^{k}$

In this case, we insert $p_{2}$ at $\left(2^{k+1}, 2^{k+1}\right), p_{3}$ at $\left(2^{k}, 0\right), p_{4}$ at $\left(0,2^{k}\right), p_{5}$ until $p_{12}$ are assigned as shown in Figure 6.

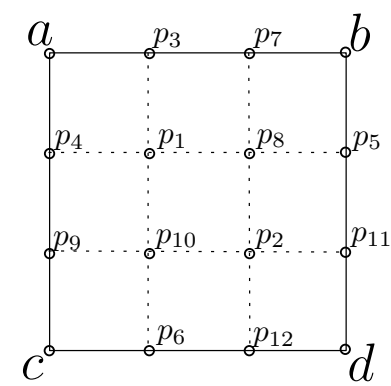

Fig. 6. Case 3 of the first phase.

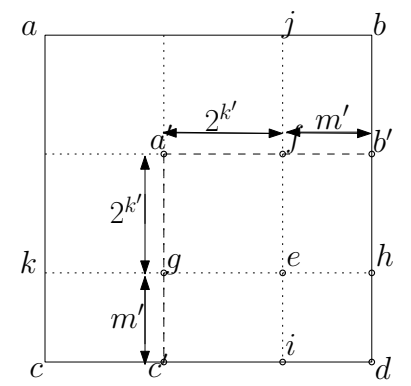

Fig. 7. Before insert points in next phase.

Let $m=(1+x) \times 2^{k}$, we have $1+\sqrt{2} / 2 \leq x<2$. Now we analyze the gap ratio after each insertion.

Lemma 3 In case 3: $(2+1 / \sqrt{2}) \cdot 2^{k} \leq m<3 \cdot 2^{k}$, the gap ratio is no more than $2 \sqrt{2}$ after each insertion according to the strategy. (Proof is in Appendix.)

After this phase, the up-left part is the combination of small square grids of size $\left(2^{k}, 2^{k}\right)$, the down-right part is a square grid of size $\left(m-2^{k+1}, m-2^{k+1}\right)$, the up-right and down-left parts are combinations of rectangles of size $\left(2^{k}, m-2^{k+1}\right)$.

\subsection{The Following Phases}

After the first phase, the square grid is partitioned into squares and rectangles. There are three types of such square or rectangle, i.e., the small square grids in the up-left part, the square grid in the down-right part, and the rectangles in the up-right and down-left parts. The only square grid in the down-right part is adjacent to the other two types of square grid and rectangle.

For clarity, we shall only consider the insertion of points in four of the squares and rectangles; specifically, the square at the down-right part, the rectangle immediately to its left, the rectangle above it and the square in the top-left part that has a common grid-position with it . Consider the configuration shown in Figure 7, we only focus on the insertion in the square grid $a^{\prime} b^{\prime} c^{\prime} d$. In such configuration, $a^{\prime}, b^{\prime}, c^{\prime}, d$ to $i$ are assigned, the down-right part is the square ehid, which is adjacent to $a^{\prime} f g e, f b^{\prime} e h$ and $g e c^{\prime} i$. If a point is to be inserted in a square grid 
or a rectangle according to the strategy, the actual processing is inserting points one by one at the same position in the square grids or rectangles of the same type until all such positions are assigned. For example, in Figure 7, there are four small square grids in the up-left part, if the strategy assigns a point in square grid $a^{\prime} f g e$, the actual assignment is inserting four points into the four small grids in square ajke.

Now we consider the insertion in configuration as shown in Figure 7. In this configuration, points $a$ to $i$ are already assigned. The up-left part is a square grid of size $2^{k^{\prime}} \times 2^{k^{\prime}}$, the down-right part is a square grid of size $m^{\prime} \times m^{\prime}$, the upright and down-left part are rectangles of size $2^{k^{\prime}} \times m^{\prime}$. When inserting the first point in the down-right square, we use the same strategy as in the first phase, i.e., find the value $k^{\prime \prime}$ such that $3 \cdot 2^{k^{\prime \prime}-1} \leq m^{\prime}<3 \cdot 2^{k^{\prime \prime}}$, then insert the point at $\left(2^{k^{\prime}}+2^{k^{\prime \prime}}, 2^{k^{\prime}}+2^{k^{\prime \prime}}\right)$.

From Case 1 of the first phase, we have $m^{\prime}=m-2^{k}, 2^{k^{\prime}}=2^{k-1}$, and $3 \cdot 2^{k-1} \leq m<(2+\sqrt{2}) \cdot 2^{k-1}$, thus, $2^{k^{\prime}} \leq m^{\prime} \leq \sqrt{2} \cdot 2^{k^{\prime}}$. From case 2 of the first phase, we have $m^{\prime}=m-2^{k}, 2^{k^{\prime}}=2^{k}$, and $(2+\sqrt{2}) \cdot 2^{k-1} \leq m<(2+1 / \sqrt{2}) \cdot 2^{k}$, thus, $\sqrt{2} \cdot 2^{k^{\prime}-1} \leq m^{\prime} \leq(1+\sqrt{2} / 2) \cdot 2^{k^{\prime}}$. From case 3 of the first phase 1 , we have $m^{\prime}=m-2^{k+1}, 2^{k^{\prime}}=2^{k}$, and $(2+1 / \sqrt{2}) \cdot 2^{k} \leq m<3 \cdot 2^{k}$, thus, $\sqrt{2} \cdot 2^{k^{\prime}-1} \leq m^{\prime} \leq 2^{k^{\prime}}$.

Combine all these cases, we have $\sqrt{2} \cdot 2^{k^{\prime}-1} \leq m^{\prime} \leq(1+\sqrt{2} / 2) \cdot 2^{k^{\prime}}$, this constraint can be relaxed to $\sqrt{2} \cdot 2^{k^{\prime}-1} \leq m^{\prime}<2 \cdot 2^{k^{\prime}}$. Let $m^{\prime}=x \cdot 2^{k^{\prime}}$, we have $\sqrt{2} / 2 \leq x<2$. Now we show how to insert points in such configuration.

Case 1: $\sqrt{2} \cdot 2^{k^{\prime}-1} \leq m^{\prime}<2^{k^{\prime}}$

In this case, we insert points $p_{1}$ until $p_{7}$ in the configuration as shown in Figure 8 .

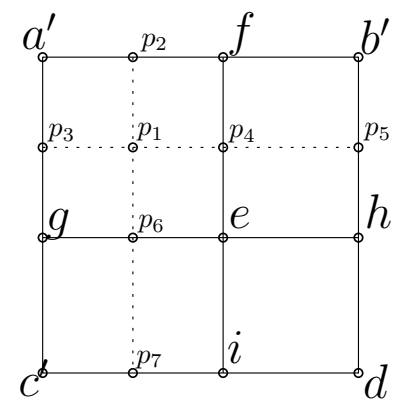

Fig. 8. Case 1 of the following phase.

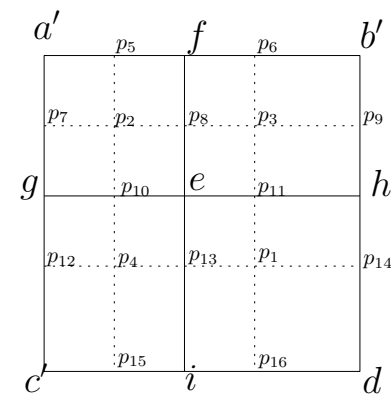

Fig. 9. Case 2 of the following phase.

After the insertion of $p_{1}$, the minimal gap is decreased to the length of $a^{\prime} p_{1}$, which is $\sqrt{2} 2^{k^{\prime}-1}$, the maximal gap is twice the length of the circumradius of the rectangle $b^{\prime} f e h$, which is $\sqrt{1+x^{2}} 2^{k^{\prime}}$. Thus, the gap ratio is at most $\sqrt{2\left(x^{2}+1\right)}$, which is no more than $2 \sqrt{2}$.

After the insertion of $p_{2}$, the minimal gap is decreased to $2^{k^{\prime}-1}$, while the maximal gap is still $\sqrt{x^{2}+1} 2^{k^{\prime}}$. Thus, the gap ratio is $2 \sqrt{x^{2}+1}$, which is no more than $2 \sqrt{2}$ since $x<1$.

After the insertion of $p_{3}$ until $p_{7}$, the minimal gap remains as $2^{k^{\prime}-1}$, and the maximal gap does not increase. Thus, the gap ratio is still no more than $2 \sqrt{2}$. 
When the insertions of this phase complete, $m^{\prime}$ remains unchanged but the size of the square grids in up-left part is decreased to $\left(2^{k^{\prime}-1}, 2^{k^{\prime}-1}\right)$. Therefore, in the next phase, the constraint of $m^{\prime}$ is $\sqrt{2} 2^{k^{\prime}} \leq m^{\prime}<2^{k^{\prime}+1}$.

Case 2: $2^{k^{\prime}} \leq m^{\prime}<(1+\sqrt{2} / 4) 2^{k^{\prime}}$

In this case, since the down-right square grid is larger, we insert points $p_{1}$ until $p_{16}$ in the configuration as shown in Figure 9 .

Note that $4 \cdot 2^{k^{\prime}-2} \leq m^{\prime}<(2+\sqrt{2} / 2) 2^{k^{\prime}-1}$, if we regard $m^{\prime}=m$ and $2^{k^{\prime}-1}=2^{k}$, this constraint is within the range in case 2 of the first phase, i.e., $(2+\sqrt{2}) \cdot 2^{k-1} \leq m<(2+1 / \sqrt{2}) \cdot 2^{k}$. Thus, similar to the analysis in case 2 of the first phase, we can say that the ratio after each insertion is no more than $2 \sqrt{2}$.

When the insertions complete, $m^{\prime}$ is decreased by $2^{k^{\prime}-1}$ and the size of the square grids in up-left part is decreased to $\left(2^{k^{\prime}-1}, 2^{k^{\prime}-1}\right)$, therefore, in the next phase, the constraint of $m^{\prime}$ is $2^{k^{\prime}} \leq m^{\prime}<(1+\sqrt{2} / 2) 2^{k^{\prime}}$.

Case 3: $(1+\sqrt{2} / 4) 2^{k^{\prime}} \leq m^{\prime}<3 \cdot 2^{k^{\prime}-1}$

In this case, we insert points $p_{1}$ until $p_{27}$ in the configuration as shown in Figure 10 .

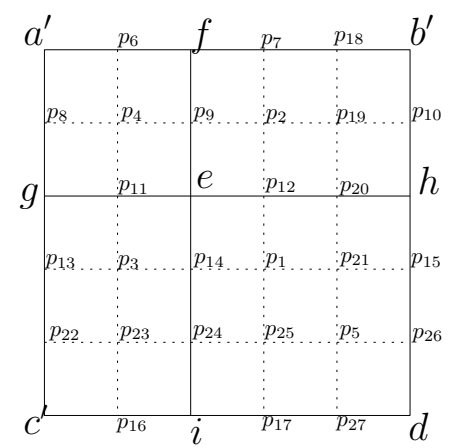

Fig. 10. Case 3 of the following phase.

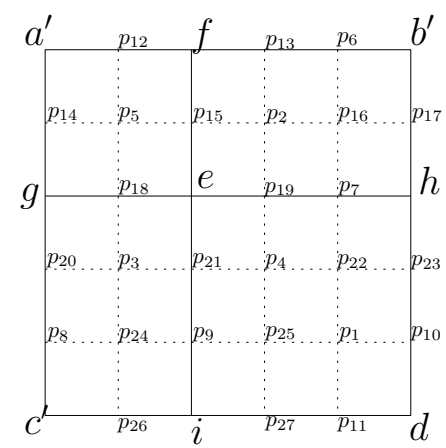

Fig. 11. Case 4 of the following phase.

If we regard $m^{\prime}=m$ and $2^{k^{\prime}-1}=2^{k}$, the constraint in this case is within the range in case 3 of the first phase, i.e., $(2+1 / \sqrt{2}) \cdot 2^{k} \leq m<3 \cdot 2^{k}$. Thus, similar to the analysis in case 3 of the first phase, we can say that the ratio after each insertion is no more than $2 \sqrt{2}$.

When the insertions complete, $m^{\prime}$ is decreased by $2^{k^{\prime}}$ and the size of the square grids in up-left part is decreased to $\left(2^{k^{\prime}-1}, 2^{k^{\prime}-1}\right)$, therefore, in next phase, the constraint of $m^{\prime}$ is $\sqrt{2} 2^{k^{\prime}-1} \leq m^{\prime}<2^{k^{\prime}}$.

Case 4: $3 \cdot 2^{k^{\prime}-1} \leq m^{\prime}<(1+\sqrt{2} / 2) \cdot 2^{k^{\prime}}$

In this case, we insert points $p_{1}$ until $p_{27}$ in the configuration as shown in Figure 11.

If we regard $m^{\prime}=m$ and $2^{k^{\prime}-1}=2^{k}$, the constraint in this case is within the range in case 1 of the first phase, i.e., $3 \cdot 2^{k-1} \leq m<(2+\sqrt{2}) \cdot 2^{k-1}$. Thus, similar to the analysis in case 1 of the first phase, we can say that the ratio after each insertion is no more than $2 \sqrt{2}$. 
When the insertions complete, $m^{\prime}$ is decreased by $2^{k^{\prime}}$ and the size of the small square grids is decreased to $\left(2^{k^{\prime}-1}, 2^{k^{\prime}-1}\right)$, therefore, in next phase, the constraint of $m^{\prime}$ is $2^{k^{\prime}} \leq m^{\prime}<\sqrt{2} \cdot 2^{k^{\prime}}$.

Case 5: $(1+\sqrt{2} / 2) \cdot 2^{k^{\prime}} \leq m^{\prime}<2^{k^{\prime}+1}$

In this case, we insert points $p_{1}$ until $p_{7}$ in the configuration as shown in Figure 12 .

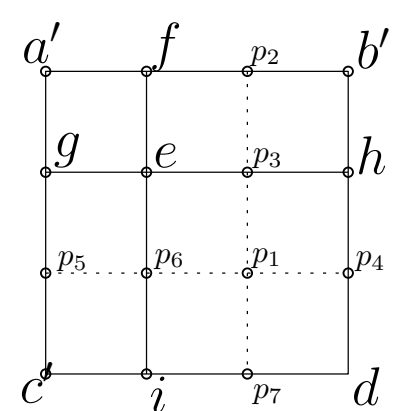

Fig. 12. Case 5 of the following phase.

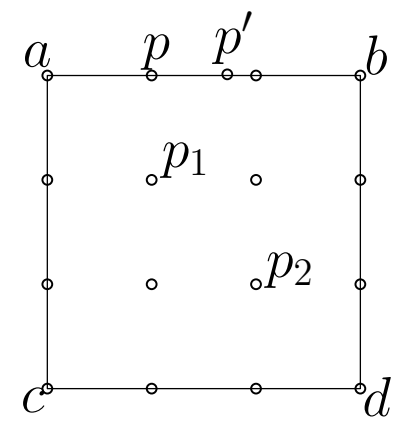

Fig. 13. In a $3 \times 3$ grid, the gap ratio is at least 2.5.

If we regard $m^{\prime}=m$ and $2^{k^{\prime}}=2^{k}$, the constraint in this case is within the range in case 2 of the first phase, i.e., $(2+\sqrt{2}) \cdot 2^{k-1} \leq m<(2+1 / \sqrt{2}) \cdot 2^{k}$. Thus, similar to the analysis in case 2 of the first phase, we can say that the ratio after each insertion is no more than $2 \sqrt{2}$.

When the insertions complete, $m^{\prime}$ is decreased to $m^{\prime}-2^{k^{\prime}}$ while the size of the square grids in up-left part remains the same as in previous phase, therefore, in next phase, the constraint of $m^{\prime}$ is $\sqrt{2} \cdot 2^{k^{\prime}-1} \leq m^{\prime}<2^{k^{\prime}}$.

Combine all these cases, we can say that the gap ratio is no more than $2 \sqrt{2}$ after each insertion. When a phase completes, suppose the size of the down-right square grid is $m^{\prime} \times m^{\prime}$, the size of the square grids in up-left part is $2^{k^{\prime}} \times 2^{k^{\prime}}$, we have $\sqrt{2} 2^{k^{\prime}-1} \leq m^{\prime}<2^{k^{\prime}+1}$.

Therefore, we have the following conclusion.

Theorem 1 The maximal gap ratio of the above strategy for inserting points into any square grid is at most $2 \sqrt{2} \approx 2.828$.

\section{Lower Bound of the Maximal Gap Ratio}

In this part, we prove that the lower bound of the maximal gap ratio is at least 2.5 for any online inserting method. Consider inserting points into a $3 \times 3$ grid, as shown in Figure 13.

initial step:

In the initial step, four corner points are already assigned. The maximal gap $G_{0}=3 \sqrt{2}$ and the minimal gap is $g_{0}=3$, thus the gap ratio at this step is $\sqrt{2}$.

inserting the first point:

If the first point is inserted at the boundary line of the square, w.l.o.g., at $p$, the maximal gap is twice the length of the circumradius of triangle $p c d$, which is 
$\sqrt{130} / 3$, the minimal gap is the length between $a$ and $p$, which is 1 , so the gap ratio is $\sqrt{130} / 3 \approx 3.8$.

If the first point is inserted at some interior point, w.l.o.g., at $p_{1}$, the gap ratio will be lower. In this case, the maximal gap is twice the length of the circumradius of triangle $b d p_{1}$, which is $\sqrt{10}$, the minimal gap is the length between $a$ and $p_{1}$, which is $\sqrt{2}$, so the gap ratio $r_{1}=\sqrt{5} \approx 2.236$.

inserting the second point:

We may assume the first point is inserted at $p_{1}$ since the gap ratio is larger if the first point is assigned at $p$.

If the second point is not inserted at $p_{2}$, the maximal gap will be $\sqrt{10}$ too, since the maximal gap must appear in triangle $b d p_{1}$ or $c d p_{1}$, and the minimal gap will be 1 , so the gap ratio $r_{2}=\sqrt{10} \approx 3.162$.

If the second point is inserted at $p_{2}$, the circumradius of triangle $b p_{1} p_{2}$ is $5 \sqrt{2} / 6$. Consider the point $p^{\prime}$ on the edge $a b$ such that $\left|p^{\prime} b\right|=5 / 4$, the length of $p^{\prime} p_{1}$ is also $5 / 4$, and the length between $p^{\prime}$ and any other point is larger than $5 / 4$. Since $5 / 4>5 \sqrt{2} / 6$, we can say the maximal gap is $5 / 2$. The minimal gap is still $\sqrt{2}$, so the gap ratio $r_{2}=5 /(2 \sqrt{2}) \approx 1.768$.

inserting the third and following points:

Similarly, we may assume the second point is assigned at $p_{2}$, otherwise the gap ratio will be larger. No matter where we assign the third point, the minimal gap will be 1 , while the maximal gap remains as $5 / 2$ owing to the symmetry of the assigned points in this square grid. Thus, the gap ratio $r_{3}=2.5$.

For the insertion of the following points, the minimal gap is 1 and the maximal gap is no larger than $5 / 2$, so the gap ratio $r_{i} \leq 2.5(i \geq 3)$.

Combining all the above cases, we can say that in handling the insertion sequence in a $3 \times 3$ square grid, the maximal gap ratio is at least 2.5 . Thus, we have the following conclusion.

Theorem 2 No online inserting method can hold the maximal gap ratio to strictly less than 2.5 .

\section{References}

1. Tetsuo Asano. Online uniformity of integer points on a line. Information Processing Letters 109(2008), pp. 57-60.

2. T. Asano, S. Teramoto. On-line uniformity of points. In: Book of Abstracts for 8th Hellenic-European Conference on Computer Mathematics and its Applications, Athens, Greece, September, 2007, pp. 21-22.

3. B.E. Bayer. An Optimum Method for Two-Level Rendition of Continuous-Tone Pictures. In Proceedings of the IEEE International Conference on Communication, pp. 11-26, 1973.

4. B. Chazelle. The Discrepancy Method: Randomness and Complexity. Cambridge University Press, 2000.

5. Charles R. Collins, and Kenneth Stephenson. A circle packing algorithm. Computational Geometry: Theory and Applications, 25(3), 2003, pp. 233-256.

6. J. Matoušek. Geometric Discrepancy. Springer, 1991.

7. Kari J. Nurmela, and Patric R. J. Östergård. More Optimal Packings of Equal Circles in a Square. Discrete \& Computational Geometry 22(3): 439-457 (1999)

8. S. Teramoto, T. Asano, N. Katoh, B. Doerr. Inserting points uniformly at every instance. IEICE Trans. Inf. Syst. E89-D (8) (2006) 2348-2356. 


\section{Appendix}

\section{Proof of Lemma 1}

Proof. After the insertion of $p_{1}$, the maximal gap is twice the length of the circumradius of triangle $a b p_{1}$, the minimal gap is the length of $p_{1} d$. Thus,

$$
\begin{gathered}
G_{1}=\frac{2(1+x) 2^{k} \times \sqrt{2} 2^{k} \times \sqrt{1+x^{2}} 2^{k}}{2(1+x) 2^{k} \times 2^{k}}=\frac{\sqrt{1+x^{2}} 2^{k+1}}{\sqrt{2}} \\
g_{1}=x \sqrt{2} 2^{k}
\end{gathered}
$$

The gap ratio at this step is $\sqrt{1+1 / x^{2}}$, which is at most $\sqrt{5}$ since $1 / 2 \leq x<$ $\sqrt{2} / 2$.

After the insertion of $p_{2}$, the minimal gap is the length of $a p_{2}$, which is $\sqrt{2} 2^{k-1}$. The maximal gap must appear in the triangle $a b p_{2}, b p_{1} p_{2}$, or $b p_{1} d$. We shall consider these three triangles respectively.

- Suppose the maximal gap appears in triangle $a b p_{2}$, since $x<\sqrt{2} / 2$, the maximal gap must be $(x+y) 2^{k+1}$, such that $(1 / 2-y)^{2}+(1 / 2)^{2}=(x+y)^{2}$. Thus, $y=\left(1 / 2-x^{2}\right) /(2 x+1)$ and $x+y=\left(x^{2}+x+1 / 2\right) /(2 x+1)$. Therefore, the gap ratio is $\frac{\sqrt{2}\left(2 x^{2}+2 x+1\right)}{2 x+1}<2 \sqrt{2}$.

- Suppose the maximal gap appears in triangle $b p_{1} p_{2}$. Since $\left|b p_{1}\right|=\sqrt{1+x^{2}} 2^{k}$, $\left|p_{1} p_{2}\right|=\sqrt{2} 2^{k-1},\left|b p_{2}\right|=\sqrt{1 / 4+(1 / 2+x)^{2}} 2^{k}$, and $\Delta_{b p_{1} p_{2}}=(1+x) 2^{2 k-2}$, the maximal gap is $\frac{\sqrt{2} \sqrt{1+x^{2}} \sqrt{1 / 4+(1 / 2+x)^{2}} 2^{k}}{(1+x)}$. Therefore, the gap ratio is $\frac{2 \sqrt{1+x^{2}} \sqrt{x^{2}+x+1 / 2}}{1+x}$, this value is strictly less than $2 \sqrt{2}$ since $1 / 2 \leq x<\sqrt{2} / 2$.

- Suppose the maximal gap appears in triangle $b p_{1} d$. Since $x<\sqrt{2} / 2<1$, the maximal gap will be $2(1 / 2+y) 2^{k}$, such that $(1 / 2-y)^{2}+1 / 4=(1 / 2+y)^{2}$. Thus, we have $y=x^{2} / 2$ and the maximal gap is $\left(x^{2}+1\right) 2^{k}$. The gap ratio is $\sqrt{2}\left(x^{2}+1\right)<2 \sqrt{2}$.

After the insertion of $p_{3}$, the maximal gap remains same as the previous step, but the minimal gap is decreased to $x 2^{k}$. Similar to above analysis,

- Suppose the maximal gap appears in triangle $a c p_{2}$, the maximal gap must be $\left.x^{2}+x+1 / 2\right) /(2 x+1) 2^{k+1}$. Therefore, the gap ratio is $\frac{2 x^{2}+2 x+1}{2 x^{2}+x}<2 \sqrt{2}$ since $1 / 2 \leq x<\sqrt{2} / 2$.

- Suppose the maximal gap appears in triangle $c p_{1} p_{2}$. The maximal gap is $\frac{\sqrt{2} \sqrt{1+x^{2}} \sqrt{1 / 4+(1 / 2+x)^{2}} 2^{k}}{(1+x)}$. Therefore, the gap ratio is $\frac{\sqrt{2} \sqrt{1+x^{2}} \sqrt{x^{2}+x+1 / 2}}{x(1+x)}$; this value is strictly less than $2 \sqrt{2}$ since $1 / 2 \leq x<\sqrt{2} / 2$.

- Suppose the maximal gap appears in triangle $c p_{1} d$. Since $x<\sqrt{2} / 2<1$, the maximal gap is $\left(x^{2}+1\right) 2^{k}$. The gap ratio is $\left(x^{2}+1\right) / x<2 \sqrt{2}$.

After the insertion of $p_{4}, p_{5}$, and $p_{6}$, the minimal gap is still $x 2^{k-1}$ but the maximal gap does not increase. Thus, the gap ratio at this step is still no more than $2 \sqrt{2}$.

After the insertion of $p_{7}$, the minimal gap is decreased to $2^{k-1}$. The maximal gap is twice the length of the circumradius of the rectangle $b p_{3} p_{1} p_{5}$, which is 
$\sqrt{x^{2}+1} 2^{k}$. Thus, the gap ratio is no more than $2 \sqrt{x^{2}+1}<2 \sqrt{2}$.

After the insertion of $p_{8}$ until $p_{12}$, the minimal gap remains as $2^{k-1}$, but the maximal gap does not increase. Thus, the gap ratio at each step is no more than $2 \sqrt{2}$.

\section{Proof of Lemma 2}

Proof. After the insertion of $p_{1}$, if $\sqrt{2} / 2 \leq x<1$, the maximal gap is twice the length of the circumradius of the triangle $a b p_{1}$, and the minimal gap is the length of $p_{1} d$; if $1 \leq x \leq 1+\sqrt{2} / 2$, the maximal gap is twice the length of the circumradius of the triangle $b p_{1} d$, and the minimal gap is the length of $a p_{1}$. Thus, we have

$-\sqrt{2} / 2 \leq x<1$

$$
\begin{gathered}
G_{1}=\frac{2(1+x) 2^{k} \times \sqrt{2} 2^{k} \times \sqrt{1+x^{2}} 2^{k}}{2(1+x) 2^{k} \times 2^{k}}=\sqrt{2\left(1+x^{2}\right)} 2^{k} \\
g_{1}=\sqrt{2} x 2^{k}
\end{gathered}
$$

The gap ratio in this case is $\sqrt{1+1 / x^{2}}$, which is at most $\sqrt{3}$.

$-1 \leq x \leq 1+\sqrt{2} / 2$

$$
\begin{gathered}
G_{1}=\frac{2(1+x) 2^{k} \times \sqrt{2} x 2^{k} \times \sqrt{1+x^{2}} 2^{k}}{2(1+x) 2^{k} \times x 2^{k}}=\sqrt{2\left(1+x^{2}\right)} 2^{k} \\
g_{1}=\sqrt{2} 2^{k}
\end{gathered}
$$

The gap ratio in this case is $\sqrt{1+x^{2}}$, which is no more than 2 .

After the insertion of $p_{2}$, the maximal gap is the same as the previous step, but the minimal gap is decreased to $x 2^{k}$ (if $\sqrt{2} / 2 \leq x<1$ ), or $2^{k}$ (if $1 \leq x \leq 1+\sqrt{2} / 2$ ). Thus, the gap ratio is $\sqrt{2\left(1+1 / x^{2}\right)}$ (if $\sqrt{2} / 2 \leq x<1$ ), which is at most $\sqrt{6}<2 \sqrt{2}$, or $\sqrt{2\left(1+x^{2}\right)}$ (if $1 \leq x \leq 1+\sqrt{2} / 2$ ), which is no more than $2 \sqrt{2}$.

After the insertion of $p_{3}$ until $p_{5}$, the minimal gap does not change, and the maximal gap does not increase. Therefore, the gap ratio after each insertion is no more than $2 \sqrt{2}$.

\section{Proof of Lemma 3}

Proof. After the insertion of $p_{1}$, the maximal gap is twice the length of the circumradius of triangle $b d p_{1}$, the minimal gap is the length of $a p_{1}$. Thus,

$$
\begin{gathered}
G_{1}=\frac{2(1+x) 2^{k} \times \sqrt{2} x 2^{k} \times \sqrt{1+x^{2}} 2^{k}}{2 x(1+x) 2^{k} \times 2^{k}}=\sqrt{2\left(1+x^{2}\right)} 2^{k} \\
g_{1}=\sqrt{2} 2^{k}
\end{gathered}
$$

The gap ratio at this step is $\sqrt{1+x^{2}}$, which is at most $\sqrt{5}$ since $1+\sqrt{2} / 2 \leq x<2$.

After the insertion of $p_{2}$, the minimal gap is the length of $d p_{2}$, which is $\sqrt{2}(x-$ $1) 2^{k}$. The maximal gap must appear in the triangle $a b p_{1}, b p_{1} p_{2}$, or $b p_{2} d$. We consider these three triangles respectively. 
- Suppose the maximal gap appears in triangle $a b p_{1}$, since $x<2$, the maximal gap must be $2(x-1+y) 2^{k}$, such that $(1-y)^{2}+1=(x-1+y)^{2}$. Thus, $y=\left(1+2 x-x^{2}\right) /(2 x)$ and $x-1+y=\left(x^{2}+1\right) /(2 x)$. Therefore, the gap ratio is $\frac{x^{2}+1}{\sqrt{2} x(x-1)}<2 \sqrt{2}$.

- Suppose the maximal gap appears in triangle $b p_{1} p_{2}$. Since $\left|b p_{1}\right|=\sqrt{1+x^{2}} 2^{k}$, $\left|p_{1} p_{2}\right|=\sqrt{2} 2^{k},\left|b p_{2}\right|=\sqrt{4+(x-1)^{2}} 2^{k}$, and $\Delta_{b p_{1} p_{2}}=(1+x) 2^{2 k-1}$, the maximal gap is $\frac{\sqrt{2} \sqrt{1+x^{2}} \sqrt{x^{2}-2 x+5} 2^{k}}{1+x}$. Therefore, the gap ratio is $\frac{\sqrt{1+x^{2}} \sqrt{x^{2}-2 x+5}}{x^{2}-1}$, this value is no more than $2 \sqrt{2}$.

- Suppose the maximal gap appears in triangle $b p_{2} d$. The maximal gap is $2(1+$ $y) 2^{k}$, such that $(1-y)^{2}+(x-1)^{2}=(1+y)^{2}$. Thus, we have $y=(x-1)^{2} / 4$ and the maximal gap is $\left(x^{2}-2 x+5\right) 2^{k-1}$. The gap ratio is $\frac{x^{2}-2 x+5}{2 \sqrt{2}(x-1)}<2 \sqrt{2}$.

After the insertion of $p_{3}$, the maximal gap remains the same as the previous step, but the minimal gap is decreased to $2^{k}$. Similar to the above analysis,

- Suppose the maximal gap appears in triangle $a c p_{1}$, the maximal gap must be $\frac{x^{2}+1}{x} 2^{k}$. Therefore, the gap ratio is $\frac{x^{2}+1}{x}<2 \sqrt{2}$.

- Suppose the maximal gap appears in triangle $c p_{1} p_{2}$. The maximal gap is $\frac{\sqrt{2} \sqrt{1+x^{2}} \sqrt{x^{2}-2 x+5} 2^{k}}{1+x}$. Therefore, the gap ratio is $\frac{\sqrt{2} \sqrt{1+x^{2}} \sqrt{x^{2}-2 x+5}}{1+x}$, this value is strictly less than $2 \sqrt{2}$.

- Suppose the maximal gap appears in triangle $c p_{2} d$. The maximal gap is $\left(x^{2}-\right.$ $2 x+5) 2^{k-1}$. The gap ratio is $\left(x^{2}-2 x+5\right) / 2<2 \sqrt{2}$.

After the insertion of $p_{4}, p_{5}$, and $p_{6}$, the minimal gap is still $2^{k}$ but the maximal gap does not increase. Thus, the gap ratios at these steps are still no more than $2 \sqrt{2}$.

After the insertion of $p_{7}$, the minimal gap is decreased to $(x-1) 2^{k}$. The maximal gap is twice the length of the circumradius of the rectangle $p_{1} p_{4} c p_{6}$, which is $\sqrt{x^{2}+1} 2^{k}$. Thus, the gap ratio is $\sqrt{x^{2}+1} /(x-1)<2 \sqrt{2}$.

After the insertion of $p_{8}$ until $p_{12}$, the minimal gap remains as $(x-1) 2^{k}$, but the maximal gap does not increase. Thus, the gap ratio after each insertion is at most $2 \sqrt{2}$. 